\title{
THE EFFECT OF MUSK EXTRACTION PROCEDURES ON THE STRESS RESPONSE OF FARMED FOREST MUSK DEER (MOSCHUS BEREZOVSKII)
}

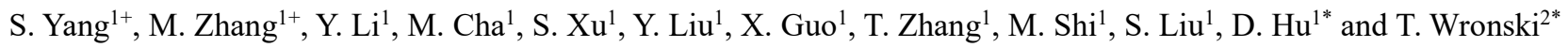 \\ ${ }^{1}$ Laboratory of Non-invasive Research Technology for Endangered Species, College of Nature Conservation, Beijing \\ Forestry University, No. 35 Tsinghua East Road, Haidian District, Beijing, 100083, China \\ 2.Faculty of Science, School of Environmental and Biological Sciences, Liverpool John Moores University, \\ Byrom Street, Liverpool L3 3AF, UK \\ *Corresponding authors Email: hudf@bjfu.edu.cn, T.Wronski@ljmu.ac.uk
}

\begin{abstract}
To determine the impact of musk extraction procedures on the endocrine stress response of farmed male forest musk deer and to improve welfare and husbandry conditions in captivity. In our study we measured the effects of musk extraction and related procedures (capture and restraint) on the stress level of treated individuals by analyzing fecal cortisol concentrations (FCC). Fecal samples were collected from 20 captive musk deer for 20 days prior and 28 days after the treatment. Ten animals were subjected to capture, restraint, and extraction (CRE-treatment), another ten experienced only capture and restraint (CR-treatment), but no extraction. Generally, stress levels observed in the CRE group were significantly higher than those of the CR group. In the CRE group, seven individuals significantly increased, but three decreased the FCC after treatment, while in the CR group, eight individuals increased and two decreased the FCC after treatment. However, the FCC recovery time in the CRE group was significantly longer than that of the CR group. Furthermore, musk deer with higher baseline levels were more likely to decrease FCC after handling. Our results suggest that individual personality, the intrinsic stress level and poor habituation to captivity permanently increase baseline FCC, leading to a sensory adaptation of the HPA axis and therefore to a decreased FCC after CRE-treatment.
\end{abstract}

Key words: Fecal cortisol concentration; Human handling; Captive breeding; Musk production; Stress hormones; Animal welfare.

https://doi.org/10.36899/JAPS.2020.6.0164

Published online August 03,2020

\section{INTRODUCTION}

Wildlife species, specifically those with high economic value, e.g., Asian black bear (Ursus thibetanus (Davis et al., 2008; Foley et al., 2011)), sika deer (Cervus nippon (Wada et al., 2007; Meng et al., 2015) ), crocodiles (Crocodylus spp) (Buenviaje et al., 1994; Revol, 1995), European mink (Mustela lutreola) (Bonesi and Palazon, 2007) or musk deer (Moschus spp) (Homes, 1999; Wu and Wang, 2006; Sheng and Liu, 2007) are increasingly farmed to satisfy consumer demands for wildlife products and to protect declining wild populations from further off-take. Theory suggests, that market-based wildlife farming - as a legal substitute for wild-hunted animals saturates the market with lawful, high-quality and affordable products, and thus facilitates conservation (Brown and Layton, 2001; Bulte and Damania, 2005; Mitra, 2005; Tensen, 2016). However, unnatural environmental conditions in captivity and routine handling procedures cause stress in animals reared on such wildlife farms (Morgan and Tromborg, 2007). In recent years, the health, welfare and well-being of captive wildlife species have received increasing attention by wildlife conservationists, animal rights activists and captive collection managers (Moberg, 1985;
Wielebnowski et al., 2002; Laule et al., 2003). Increased stress responses are hereby considered the key problem (Thompson, 1989; Becker and Lobato, 1997; Meng et al., 2010; Meng et al., 2011b), affecting the physical and mental health of farmed animals (McEwen, 2004; Teixeira et al., 2007) and leading to immunity dysregulations (Dhabhar and McEwen, 1997; Gouin et al., 2008). To evaluate and mitigate the adverse effects of stress in captive wildlife species becomes thus increasingly important (Weinberg and Levine, 1980; Hemsworth et al., 1987; He et al., 2014).

Musk deer are small solitary ungulates and belong to a distinctive taxonomic family, the Moschidae, phylogenetically related to bovids rather than cervids (Hassanin et al., 2012). Musk deer are well known for the musk, a viscous substance secreted by specific glands located between the navel and the genitals of males (Homes, 1999; Wu and Wang, 2006; Sheng and Liu, 2007). For more than 5,000 years, musk is used for the production of medicine in East Asian countries, but also as a scent fixative in perfumes and other cosmetics (Pilz, 1997; Homes, 1999). Wild populations of musk deer have experienced an over-exploitation across their natural range resulting together with habitat loss in a drastic population decline in recent decades (Homes, 1999; Yang 
et al., 2003). All musk deer species are listed in Appendix I or II of the Convention on International Trade in Endangered Species of Wild Fauna and Flora (UNEPWCMC, 2014), and-apart from the Siberian musk deer, M. moschiferus - are considered 'Endangered' by the IUCN Red List (IUCN, 2018).

Traditionally, musk was harvested by hunting and killing wild males (Yang et al., 2003), but due to intensive research by scientists and farming practitioners, methods were developed to obtain musk from live animals (Shrestha, 1989; Wu and Wang, 2006; Kang et al., 2008; Zhang et al., 2009). The new extraction method not only involves the capture and restraint of suitable males, but also requires an operator with a sterilized spatula to extract musk from the musk sac, a pouch-like invagination of glandular tissue (Zhang, 1983). Musk production in male musk deer is highest at the age of 1.5 to 7.5 years, and the annual average musk extraction ranges from $10.72 \mathrm{~g}$ to $13.53 \mathrm{~g}$ per individual (Cheng et al., 2002). In China, musk is currently traded at around 45,000 US\$ per kilogram, which makes it more precious than gold (currently about 38,000 US\$ per kilogram; (Wang et al., 2015). Since the beginning of musk deer farming in the 1950s, the new extraction method became increasingly established and is nowadays widely accepted and applied (Shrestha, 1998; Homes, 1999; Parry-Jones and Wu, 2001). Various sources (Shrestha, 1998; Homes, 1999; Parry-Jones and $\mathrm{Wu}, 2001)$ reported that musk extraction does not impede or harm the male's growth, reproduction or health. However, in practice, unskilled extraction of musk can cause necrosis of the musk sac tissue, reduced musk secretion, fierce stress responses and in some cases fatality (Shrestha, 1998; Wu and Wang, 2006; Meng et al., 2011a). Musk deer are shy, timid, and very attentive and vigilant to human approach $(\mathrm{Wu}$ and Wang, 2006). In addition, extraction procedures are rather invasive, including capture, restraint or immobilization and extraction, the opening of the musk sac, and the physical removal of dried secretions. Each of these handling procedures directly impairs the musk deer's body and typically leads to a pronounced stress response. Surprisingly, no study has yet reported on the effects this physical extraction procedure has on the endocrinology of farmed musk deer. Stress is a key factor when evaluating animal welfare in captivity (Morgan and Tromborg, 2007; Davis et al., 2008), and glucocorticoid (GC) levels are commonly considered the most effective index to measure the degree of stress in wildlife species (Möstl and Palme, 2002). GCs contain mainly cortisol and corticosterone, both of which are commonly measured to quantify the stress response in various wildlife and domestic livestock species. Previous studies highlighted that human handling such as catching, transportation, anesthesia, or electro-ejaculation change the GC level in the feces of handled animals (Crookshank et al., 1979; Smith et al., 1999; Palme et al., 2000;
Morato et al., 2004; Orihuela et al., 2012), making fecal glucocorticoid sampling the ideal, non-invasive method to measure stress in wild and captive wildlife species (Romero and Reed, 2005).

In this study we determined whether musk harvest (including capture, restraint and musk extraction) from farmed male forest musk deer (Moschus berezovskii) triggered higher stress responses, i.e. higher fecal cortisol concentrations (FCC) than capture and restraint alone. To test this we subjected ten males to the extraction procedure (including capture, restraint and musk extraction; CRE-treatment) and another ten animals to only capture and restraint (CR-treatment). We tested the following predictions:

i) We expected the baseline concentration, i.e. the FCC prior to any treatment to be equal in both treatment groups. ii) Previous studies of FCC in other species (Cyr and Michael Romero, 2007; Linklater et al., 2010) indicated that stress responses differ between individuals and that FCC occasionally decreased after treatment. We therefore predicted that both, CRE- and CR-treatment could cause a change in FCC in either direction but that this change will be more pronounced in the CRE-treatment group than in the CR-treatment group. iii) The FCC recovery time after treatment will be longer in the CRE- than in the CR-treatment group. iv) The recovery time will also differ between individuals with increased FCC and those with decreased FCC whereby it was expected that individuals with increased FCC have a longer recovery time. v) The variation of FCC will be higher in the CRE-treatment group than in the CRtreatment group. vi) Variation of FCC will be higher in individuals that increased their FCC after treatment than in individuals that decreased their FCC after treatment. vii) Eventually, we asked whether an increase or decrease in FCC was depending on the individuals' baseline FCC prior to handling.

\section{MATERIALS AND METHODS}

Study site and animals: The study was conducted in the Pien Tze Huang Forest Musk Deer Breeding Center located in Fengxian, Shaanxi Province, at the northern site of the Qinling Mountains in China $\left(34^{\circ} 16^{\prime} 40.16^{\prime \prime} \mathrm{N}\right.$, $\left.106^{\circ} 47^{\prime} 03.08^{\prime \prime} \mathrm{E}\right)$. The climate is warm and temperate, with an average temperature of $11.4^{\circ} \mathrm{C}$ and an annual rainfall of 613.2 to $897.1 \mathrm{~mm}$. The breeding center was established in a remote, rural area, mainly for commercial purpose (but also for research), providing space for more than 300 forest musk deer in 60 breeding units. Each breeding unit consists of ten wall-fenced compartments $\left(2 \times 2 \times 3.5 \mathrm{~m}^{3}\right)$ and one $10 \times 8 \mathrm{~m}$ open-air activity space sealed by wire mesh to prevent escape through the top. Due to the pronounced territoriality in male musk deer (Wu and Wang, 2006; Sheng and Liu, 2007), each compartment contains only one adult animal older than 
one year. Males and females are kept in separate compartments and meet only for mating. The musksecreting period of male forest musk deer in the Qinling Mountains reaches from May to the end of July (breeding season). Musk is therefore harvested at least two to three months after this period, i.e. during the non-breeding season when secretions desiccated inside the musk sac (Wu and Wang, 2006; Sheng and Liu, 2007). Animals were fed twice a day, mainly with fresh leaves (in summer and autumn) or dried leaves (in winter and spring) originating from the natural habitat of wild musk deer. Major food plants include Chinese sumac (Rhus spec., Anacardiaceae), bitterwood (Picrasma spec., Simaroubaceae), elm (Ulmus pumila), as well as some supplementary food, consisting of flour, wheat bran and some vegetables of the season. Water was provided ad libitum and all animals were kept in the same pathogenfree environment.

Fecal Sample Collection: The study subjects consisted of twenty healthy adult male musk deer aged two to eight years of which 10 individuals underwent capture, restraint, and extraction (CRE-treatment), while another ten were only subjected to capture and restraint, but not to extraction (CR-treatment). Sample collection was conducted between August and October. Prior to treatment fecal samples were taken every two days over a period of 20 days (app. $10 \mathrm{~g}$ per sampling). After treatment, fecal samples were collected every day for a period of 10 days, followed by a period of 18 days during which samples were taken every second day (app. 10g per sampling). Fecal samples were collected in the early morning hours (between 6:00 and 8:00 am), and stored frozen at $-20^{\circ} \mathrm{C}$ immediately after collection. Male musk deer defecate 3-6 times per day (i.e., in 24 hours), suggesting that fecal samples were not older than eight hours when collected in the morning. After that time the stability of the cortisol in the fecal sample was still sufficiently high for laboratory determination of the FCC (Wasser et al., 1988; Lang et al., 2013).

Capture, restraint and extraction: The capture and restraint procedures were carried out as follows: the male musk deer was expelled from his compartment into a wooden box measuring $80 \times 20 \times 60 \mathrm{~cm}^{3}$. Opening the box from the rear side, an animal keeper grabbed the hind legs, pulling out the animal while quickly grabbing the fore legs. The male was then laid on its back across the legs of a sitting keeper. Two additional persons assisted, one by constraining the rear legs of the animal, another one holding forelegs and head to prevent potential injuries caused by the fangs. Musk extraction was conducted by a specialized veterinarian who inserted a spatula through the orifice into the musk sac to extract the musk (Fig. 1). Due to the limited size of the orifice, this action was repeated several times. After extraction was completed, the orifice of the musk sac was treated with antibiotic ointment. The handling time for each animal was limited to 12 minutes, meaning the animal was released, even though the extraction was not terminated. All CR-treatment animals were subjected to the same procedures, except that no spatula was inserted into the musk sac and no musk was extracted.

Fecal cortisol extraction and determination: The individual fecal cortisol concentration (FCC) was determined as described in Isobe et al. (2005) and Capezzuto et al. (2008) with minor modifications(Isobe et al., 2005; Capezzuto et al., 2008) . In brief, undried feces $(0.5 \mathrm{~g} \pm 10 \mathrm{mg})$ were weighed and placed in a $15 \mathrm{ml}$ centrifuge vial. $5 \mathrm{ml}$ of $90 \%$-ethanol were added and incubated for 20 minutes. Subsequently, the mixture was centrifuged at $2500 \mathrm{rpm}$ for 20 minutes. The supernatant was then transferred to a capped vial, another $5 \mathrm{ml}$ of $90 \%$ ethanol was added to the $15 \mathrm{ml}$ centrifuge vial, followed by one minute vortexing and centrifugation at $2500 \mathrm{rpm}$ for 15 minutes. Both supernatants were recovered and combined, dried and re-dissolved in $1 \mathrm{ml}$ methanol, the solution was vortexed for one minute and stored at $-20^{\circ} \mathrm{C}$ until assayed. Simultaneously, the water content of fecal samples was calculated (after drying fecal samples in an oven at $60^{\circ} \mathrm{C}$ for eight hours), and used to adjust the final fecal steroid concentration. To determine the final FCC, Enzyme-Linked Immunosorbent Assays (ELISA) were applied. Prior to conducting ELISA tests, the methanol solution was thawed and diluted by 1:50 in phosphate buffer saline (PBS). The cortisol antibody and horseradish peroxidase were produced in CalBioreagent (Cat. No. M344 and C133). Cross reactivity of the antibody with other steroids was low (11Deoxycortisol: $\quad 0.9 \%, \quad$ Prednisolone: $\quad 5.6 \%$, Corticosterone: $0.6 \%$, 11-Deoxycorticosterone: $<0.1 \%$, Progesterone: $<0.1 \%$, 17-hydroxyprogesterone: $<0.1 \%$, Testosterone: $<0.01 \%$, Estradiol: $<0.1 \%$, Estriol: $<0.1 \%$, and Danazol: $<0.01 \%$ ). The range of the assay was estimated as $15.6-1,000 \mathrm{pg} / \mathrm{ml}$. Intra- and inter-assay coefficient of variance (CV) were $<8 \%$ and $<10 \%$, respectively.

Data analysis: In a first step we established the fecal cortisol (FC) baseline concentration, i.e., the 20 days average $\mathrm{FCC}$ for each individual before treatment (10 days $\times 10$ animals $=100$ samples per treatment). Individual baseline means were calculated using an iteration process by which all peak values with $2 \mathrm{SD}$ above the mean were excluded. Mean values were recalculated until all extreme values were omitted (Moreira et al., 2001; Yu et al., 2011). Subsequently, we tested for differences in the FC baseline concentrations between CRE- and CR- treatment. In a second step we tested whether the increased (or decreased) FCC after treatment differed between the CRE- and the CRtreatment groups (compared to baseline concentration). Individuals with lowered FCC for three consecutive days 
after treatment were regarded as having decreased FCC, while all others were classified as having increased FCC after treatment. We also tested for differences in the recovery time between CRE- and CR-treatment groups and between individuals with increased FCC after treatment and those that decreased the FCC after treatment. We further established the FCC variation range from the highest FCC to the lowest for both treatment groups. Subsequently, we tested for differences in the variation between CRE- and CR-treatment groups and between individuals that increased the FCC after treatment and those that decreased the FCC after treatment. Eventually, we tested whether individuals with increased FCC after treatment (including CRE- and CRtreatment) differed in their baseline FCC from those individuals with decreased FCC. Prior to statistical analysis, all data sets were tested for normality (Kolmogorov-Smirnov test) and homogeneity of variances (Levene's test). Accordingly, we used either an independent samples t-test or a Mann-Whitney $U$ test to test differences between variables. Results were considered significant if $P \leq 0.05$. Statistical analyses were conducted using SPSS Version 22.0 and Origin 8.0 for Windows.

\section{RESULTS}

The average baseline cortisol level of the CRtreatment group $(\mathrm{N}=10)$ was $47,326.7 \pm 3,000.4 \mathrm{pg} / \mathrm{g}$ feces and 47,769.9 $\pm 3,805.0 \mathrm{pg} / \mathrm{g}$ feces in the CREtreatment group $(\mathrm{N}=10)$. As expected, there was no significant difference between the two groups (Independent samples t-test: $t_{18}=-0.091, P=0.928$ ). Following treatments, the mean FCC of the CREtreatment group was $54,432.6 \pm 1,041.3 \mathrm{pg} / \mathrm{g}$ feces (max. FCC: $129,009.5 \mathrm{pg} / \mathrm{g}$ feces), while that of the CRtreatment group was 48,163.4 $\pm 663.3 \mathrm{pg} / \mathrm{g}$ feces (max. FCC: $80,620.8 \mathrm{pg} / \mathrm{g}$ feces). This increase of FCC confirms that both, the CRE- and the CR-treatment groups, showed hormonal stress responses after handling (Table 1).

After different treatments, the FCC measured in the CRE-treatment group increased more than that the rise observed in the CR-treatment group (Mann-Whitney $\mathrm{U}$ test: $\mathrm{Z}=-4.79, \mathrm{P}<0.01$ ). Having a closer look at the data, fifteen individuals significantly increased their FCC during the first few days after treatment. Out of these, seven individuals were subjected to CRE-treatment (Fig. 2A) and eight individuals were subjected to CR-treatment (Fig. 2C) (CRE baseline: $41745.8 \pm 3269.8 \mathrm{pg} / \mathrm{g}$ feces, CRE after treatment [4 days]: $66109.2 \pm 4139.3 \mathrm{pg} / \mathrm{g}$ feces, Independent samples t-test: $t_{33}=-2.861, P<0.01$;
CR baseline: $43692.4 \pm 2243.5 \mathrm{pg} / \mathrm{g}$ feces, CR after treatment [3 days]: $54594.7 \pm 2148.4 \mathrm{pg} / \mathrm{g}$ feces, Independent samples t-test: $t_{30}=-2.750, P<0.05$ ), but thereafter the FCC of these fifteen individuals gradually declined to baseline concentration. Compared to the baseline FCC, three individuals subjected to CREtreatment (Fig. 2B) and two individuals subjected to CRtreatment (Fig. 2D) significantly decreased the FCC after treatment (CRE baseline: $61825.9 \pm 1095.6 \mathrm{pg} / \mathrm{g}$ feces, CRE after treatment [4 days]: $53415.9 \pm 1000.5 \mathrm{pg} / \mathrm{g}$ feces, Mann-Whitney $U$ test: $Z=-2.052, P<0.05$; CR baseline: $61863.8 \pm 96.3 \mathrm{pg} / \mathrm{g}$ feces, CR after treatment [3 days]: $51146.16 \pm 1328.7 \mathrm{pg} / \mathrm{g}$ feces, Mann-Whitney $U$ test: $Z=-1.862, P=0.063$ ).

Comparing the time (days) during which the FCC was above (or below) the mean $\pm 2 \mathrm{SD}$, indicates that the FCC recovery time (mean $\pm \mathrm{SD}$ ) under CREtreatment lasted significantly longer than under CRtreatment (CRE: $10.60 \pm 1.21$ days; CR: $3.90 \pm 0.90$ days, Independent samples t-test: $t_{18}=4.436, P<0.01$ ). In the CRE-treatment group, seven individuals with increased FCC required a significantly longer recovery time than the three individuals with decreased FCC (increased FCC group: $12.43 \pm 0.53$ days, decreased FCC group: $6.33 \pm 2.67$ days, Mann-Whitney $U$ test: $Z=-$ 2.438, $P<0.05$; Fig. 3). However, in the CR-treatment group, no difference was found between the eight individuals with increased FCC and the two individuals with decreased FCC (increased FCC group: $3.75 \pm 1.13$ days, decreased FCC group: $4.50 \pm 0.50$ days, Independent samples t-test: $t_{8}=-0.32, P=0.76$; Fig. 3 ).

Post-treatment FCC variation (mean $\pm \mathrm{SD}$ ) in the CRE-treatment group was significantly higher than in the CR-treatment group (CRE: $42175.15 \pm 6069.11 \mathrm{pg} / \mathrm{g}$ feces, CR: $27154.92 \pm 1856.14 \mathrm{pg} / \mathrm{g}$ feces, Independent sample t-test: $t_{18}=2.37, P<0.05$ ). In the CRE-treatment group, seven individuals with increased FCC showed significantly higher variation than the three individuals with decreased FCC (increased FCC group: $48432.54 \pm$ $7447.83 \mathrm{pg} / \mathrm{g}$ feces, decreased FCC group: $27574.57 \pm$ $3456.84 \mathrm{pg} / \mathrm{g}$ feces, Mann-Whitney $U$ test: $Z=-2.17, P<$ 0.05). However, in the CR-treatment group, the eight individuals with increased FCC had a similar FCC variation as the two individuals with decreased FCC (increased FCC group: $26481.08 \pm 2243.59 \mathrm{pg} / \mathrm{g}$ feces, decreased FCC group: $29850.30 \pm 2238.80 \mathrm{pg} / \mathrm{g}$ feces, Independent sample t-test: $t_{8}=-0.71, P=0.50$; Fig. 4). The five individuals with decreased FCC had a significantly higher baseline FCC $(61841.10 \pm 600.96$ $\mathrm{pg} / \mathrm{g}$ feces) compared to the fifteen individuals with increased FCC $(42784.00 \pm 1883.60 \mathrm{pg} / \mathrm{g}$ feces, MannWhitney $\mathrm{U}$ test: $Z=-3.27, P<0.01)$. 


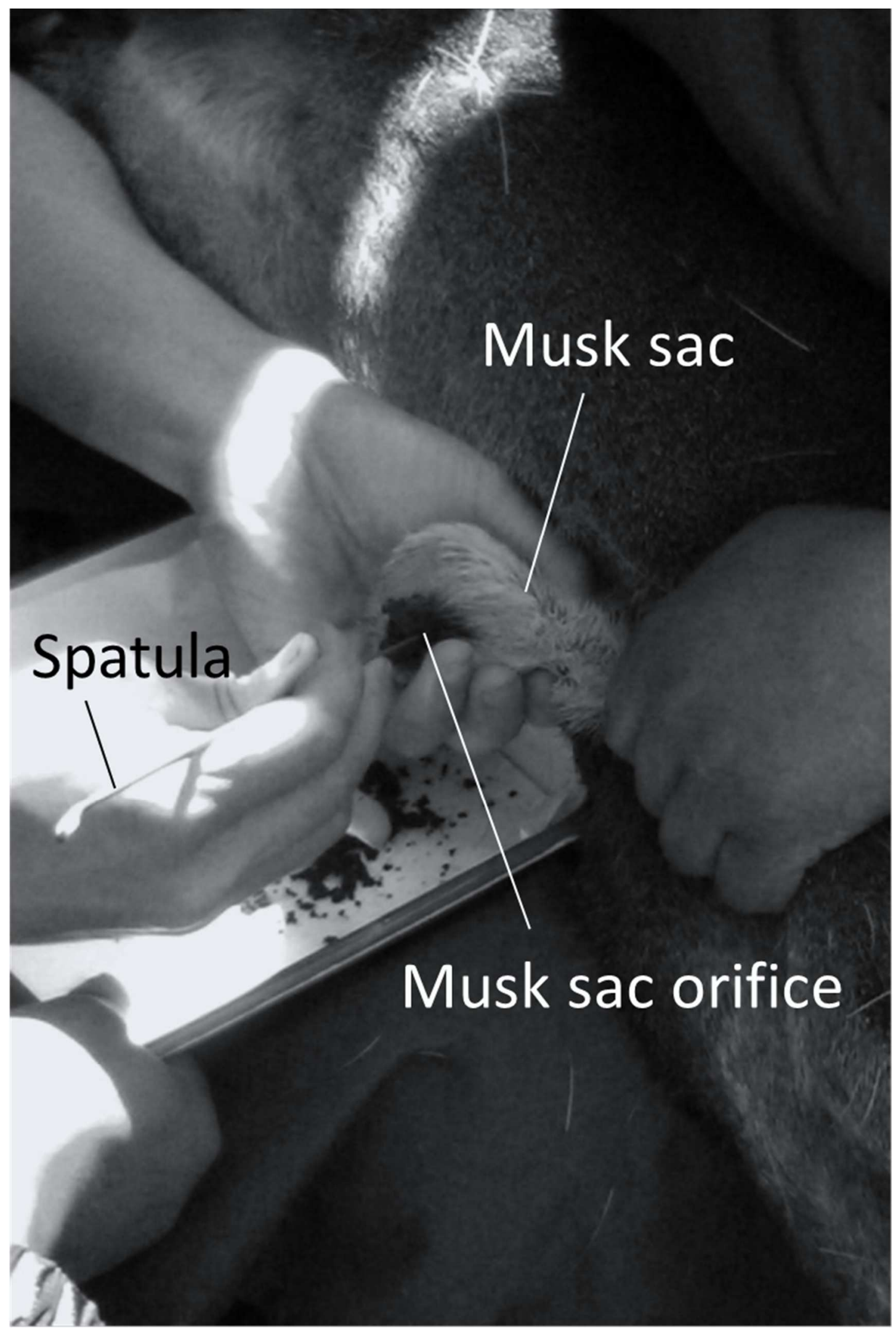

Figure 1. Extraction of musk from a living male forest musk deer (Moschus berezovskii) carried out by a specialized veterinarian and the help of two animal keepers. 

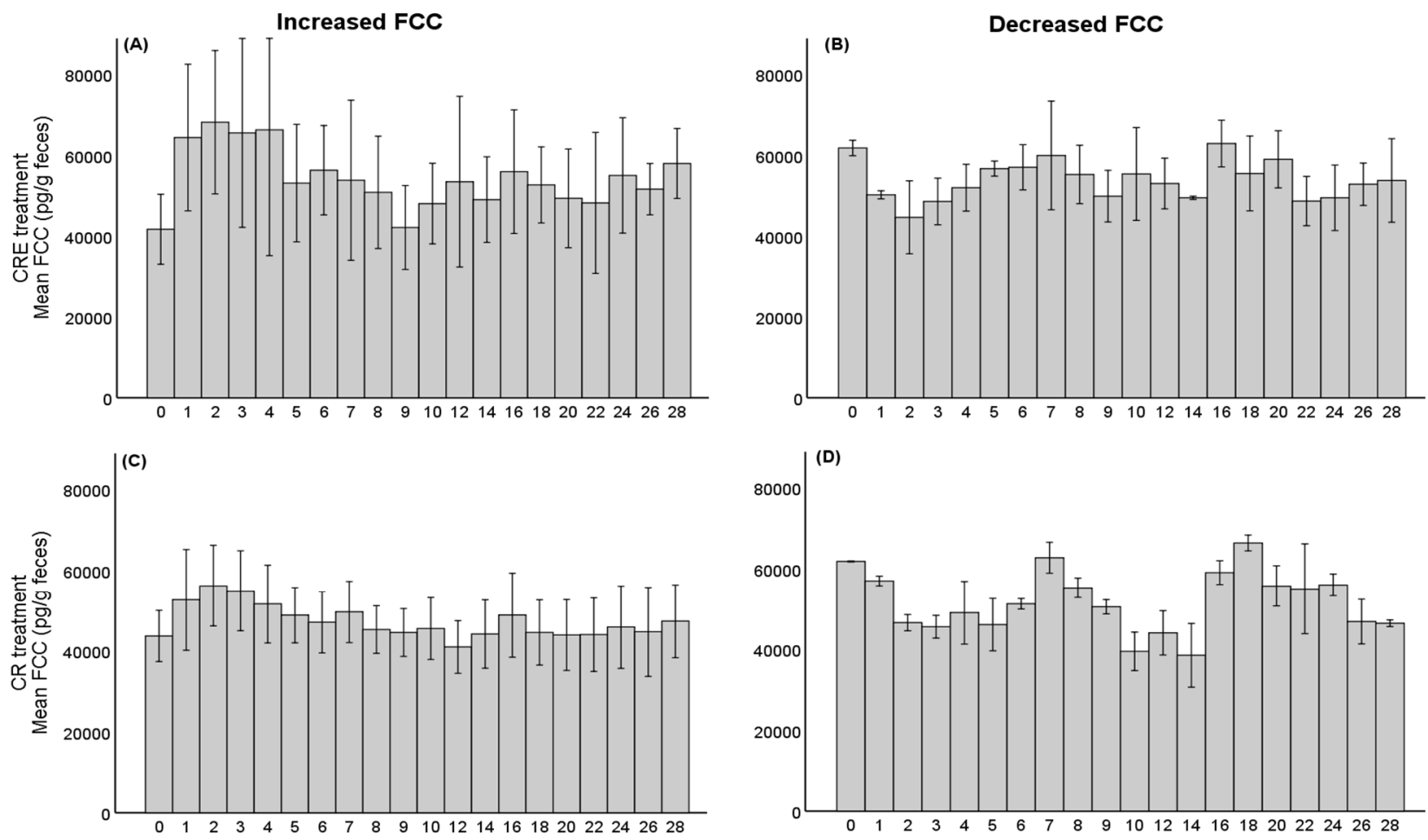

Days after treatment

Figure 2. Mean FCC progression in male musk deer after CRE- (A, B) and CR-treatment (C, D). Seven individuals significantly increased FCC during the first four days after CRE-treatment (A), while in three other animals FCC significantly decreased after the treatment (B). In eight individuals FCC significantly increased during the first three days after CR-treatment (C), while in two other individuals FCC significantly decreased after treatment (D). Note that day ' 0 ' represents the baseline FCC before treatment.

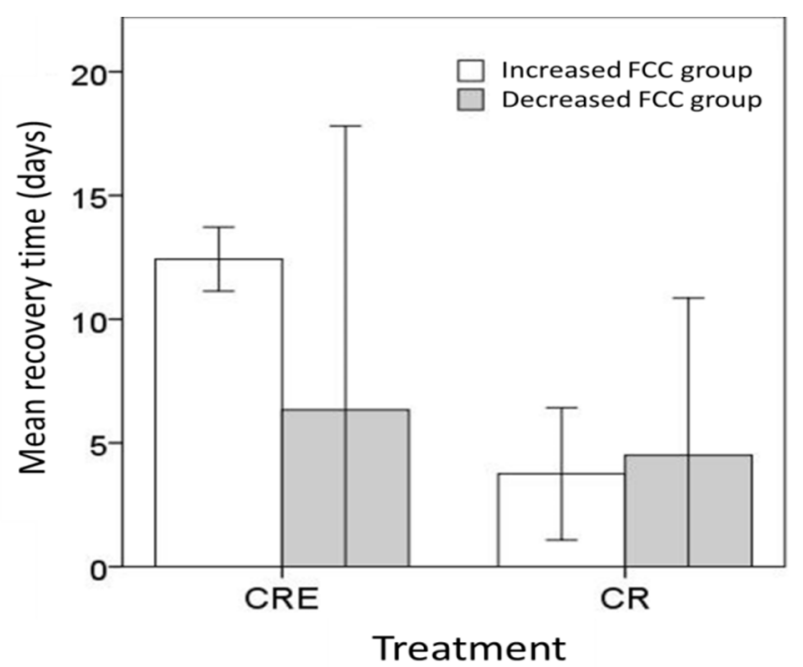

Figure 3. Mean recovery time during which the FCC was above (or below) the mean \pm 2 SD for individuals with increased and decreased FCC in both treatment groups (CRE- and CRtreatment).

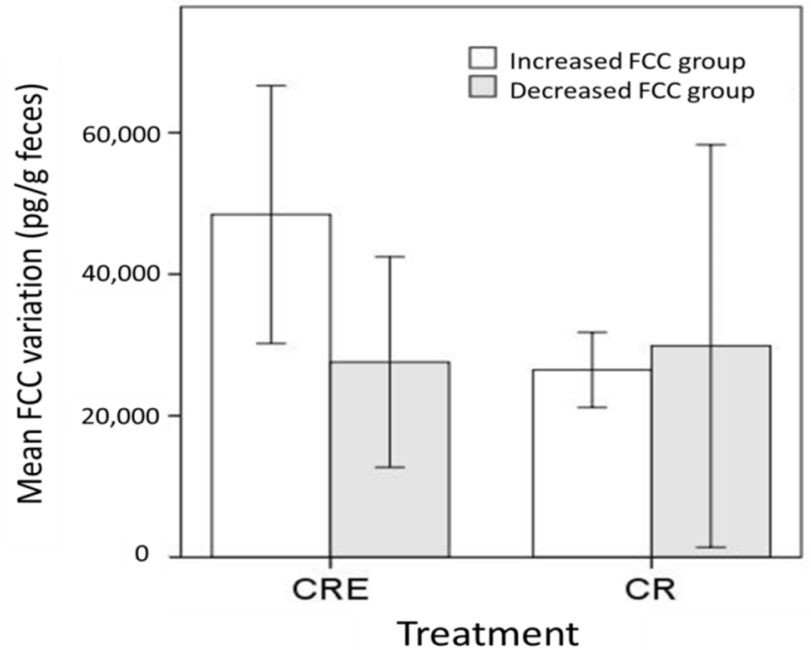

Figure 4. Post-treatment variation in the FCC for individuals with increased and decreased FCC in both treatment groups (CRE- and CRtreatment). 
Table 1. Individual baseline concentration, maximum and minimum FCC after CRE- or CR-treatment.

\begin{tabular}{|c|c|c|c|c|c|}
\hline & Individual & $\begin{array}{l}\text { Baseline FCC } \\
\text { (pg/g) }\end{array}$ & $\begin{array}{l}\text { max. FCC } \\
\text { after treatment } \\
(\mathrm{pg} / \mathrm{g})\end{array}$ & $\begin{array}{l}\text { min. FCC } \\
\text { after treatment } \\
(\mathrm{pg} / \mathrm{g})\end{array}$ & $\begin{array}{l}\text { Cortisol } \\
\text { response }\end{array}$ \\
\hline \multirow[t]{10}{*}{ CRE } & 1 & 29888.8 & 58894.0 & 22102.0 & increased \\
\hline & 2 & 34460.7 & 65353.7 & 33654.5 & increased \\
\hline & 3 & 36837.0 & 80796.4 & 38794.6 & increased \\
\hline & 4 & 40975.5 & 89247.4 & 43088.3 & increased \\
\hline & 5 & 47546.7 & 129009.5 & 39042.8 & increased \\
\hline & 6 & 48413.4 & 94792.5 & 40442.3 & increased \\
\hline & 7 & 54098.7 & 84641.3 & 46582.5 & increased \\
\hline & 8 & 60264.7 & 68534.9 & 44476.3 & decreased \\
\hline & 9 & 61274.9 & 68881.6 & 34393.7 & decreased \\
\hline & 10 & 63938.2 & 67265.5 & 43088.3 & decreased \\
\hline \multirow[t]{10}{*}{ CR } & 1 & 33318.0 & 52655.1 & 27287.0 & increased \\
\hline & 2 & 39756.0 & 66929.3 & 34835.3 & increased \\
\hline & 3 & 40871.9 & 58648.8 & 32077.0 & increased \\
\hline & 4 & 42924.0 & 62710.0 & 38851.5 & increased \\
\hline & 5 & 44192.5 & 54534.9 & 34526.0 & increased \\
\hline & 6 & 46264.7 & 68007.0 & 36111.1 & increased \\
\hline & 7 & 47018.8 & 53434.9 & 36645.9 & increased \\
\hline & 8 & 55193.3 & 80620.8 & 45358.4 & increased \\
\hline & 9 & 61767.5 & 67790.7 & 40179.2 & decreased \\
\hline & 10 & 61960.2 & 65016.5 & 32927.4 & decreased \\
\hline
\end{tabular}

\section{DISCUSSION}

Extraction of musk from living musk deer is essential to supply legal markets and to satisfy consumer demands, while at the same time benefitting the conservation of wild musk deer (Wu and Wang, 2006). Musk harvest is usually conducted once a year, inevitably causing discomfort for the treated animal. No study has yet reported on the potential adverse effects of musk extraction on the behavior and physiology of male musk deer. In this study, endocrinology of the short-term effects of CRE- vs CR-treatment were investigated by measuring fecal cortisol levels. In most cases the FCC significantly increased after CRE- and CR-treatment, verifying that human handling indeed inflicts stress responses on musk deer (Table 1). Stress responses due to human handlings were also reported from domestic animal species such as pig (Sus scrofa)(Prunier et al., 2005), sheep (Ovis aries)(Hargreaves and Hutson, 1990) and cattle (Bos taurus)(Palme et al., 2000), or from captive wildlife species like Fijian ground frog (Platymantis vitiana) (Narayan and Hero, 2011) and cheetahs (Acinonyx jubatus) (Wells et al., 2004). Apart from human handling, the solitary, timid and vigilant character of forest musk deer contributes to the increasing FCC. Musk deer retain these traits although they were held in captivity for generations (Wu and Wang, 2006; Sheng and Liu, 2007).

In our study we could show, that different handlings can cause different stress levels or stress response. With regard to the overall FCC (Table 1), the recovery time (Fig. 3 ) and the FCC variation (Fig. 4), the CRE-treatment had generally a stronger effect on male musk deer than the CR-treatment, although no differences in the initial stress level were observed. Stronger stress responses after CRE-treatment were owed to the anatomy of the musk gland. This essential organ produces the musk, an important sexual ecto-hormone used to mark the territory and to attract females during the mating season (Wu and Wang, 2006; Sheng and Liu, 2007). When excreted, fresh musk is a viscose, ropy paste, but after two to three months of drying inside the musk sac it turns into a brown, solid, waxy substance attached to the wall of the musk sac. During extraction a spatula is repeatedly inserted through the orifice to scrape off the dry musk from the wall of the musk sac (Fig. 1). The wall comprises of delicate tissue pervaded with numerous small blood vessels and nerves (Wu and Wang, 2006), far more sensitive than other body parts impaired during capture or restrain. The aggravating stimulation of this sensitive tissue activates the hypothalamus pituitary adrenocortical (HPA) axis and thus increases the cortisol production more than during CR-treatment. Studies on domestic ungulates reported similar results suggesting that different handling procedures lead to different stress responses. For example, during the well-studied procedure of sheep shearing, only the shearing itself elicited an increase of the plasma cortisol concentration while capture and restrain did not lead to increased stress responses (Pierzchala et al., 1983). The authors showed 
that the same handling procedure caused different individual stress responses, largely depending on the individuals' personality and on its physiological stress status prior to handling (Kralj-Fišer et al., 2009). Although our study confirmed these findings, five individuals did not increase their FCC after treatment, but significantly decreased the FCC (Fig. 2B, D). Moreover, individuals with decreased FCC after CRE-treatment had shorter recovery times (Fig.3) and smaller FCC variation than individuals with increased FCC after treatment (Fig.4). This pattern, however, was not apparent in the CR-treatment group, with no significant difference between increased and decreased FCC (Figs. 3, 4). Since the CRE-treatment was a stronger stressor to male musk deer than the CR-treatment, the obvious difference between individuals with increased and decreased FCC became more apparent. Meanwhile, the HPA axis in individuals with increased FCC was more inclinable and sensitive than in individuals with decreased FCC, causing individuals with increased FCC to have longer recovery times and higher FCC variation than individuals with decreased FCC. Most noteworthy is that individuals with decreased FCC had a higher initial stress level than those with increased FCC. The physiological mechanisms behind stress responses are still under debate. Several studies confirmed that an increased FCC reflects negative stress responses (Wielebnowski et al., 2002; Nephew and Romero, 2003), while the decline of FCC was interpreted rather as an acclimation process (Millspaugh et al., 2007; Franceschini et al., 2008). Miller et al. (2007) suggested that chronic stress can both increase and decrease the activity of the HPA axis and thus increase or lower the FCC. This depends mainly on the course and the duration of the stress stimulus as well as on the baseline FCC (Miller et al., 2007). In this study, the opposing direction of the HPA axis activity can be most likely attributed to different initial stress levels prior to the treatment. Individuals that increased FCC after treatment may have been better habituated to the captive surroundings, so their lower FCC baseline levels increased when they were subjected to abrupt CRE- or CR-treatments. Individuals that decreased FCC after treatment had higher baseline concentrations due to maladaptation to captivity or their more timid and vigilant personality. A new or strong stressor such as the CRE- and CR-treatments resulted in an intense stress stimulus, but due to the sensory adaptation of the HPA axis the intensity of the stress response was inhibited and the FCC even decreased. After seven to nine days though, the HPA axis regained reactivity and the FCC increased back to baseline level.

Nowadays, the extraction of musk from living animals - including related procedures such as capture and restraint - are common routine. In order to minimize welfare issues and to improve the health of captive musk deer, it is imperative to apply the best code of practice. This study is the first systematic approach, quantifying the stress responses of male musk deer to musk extraction procedures and, based on that, provides the following facts and recommendations to improve the wellbeing of musk deer in captivity:

(1) Musk extraction and related handlings have both a strong effect on the stress response of male musk deer. However, this response is less pronounced if only capture and restrain are performed.

(2) The first four to six days after treatment represent the period with the most intensive stress response, suggesting that musk deer should be held under strict silence and privacy during this period-food and water ought to be provided before returning to their compartment.

(3) The direction of the stress response depends on the personality and thus on the baseline FCC of each individual. However, individuals with decreased FCC after treatment recover faster and show less variation than individuals with increased FCC.

(4) New born males should undergo a wellstructured habituation process as suggested by (Wang et al., 2016). This will help avoiding maladaptation to captivity and high permanent baseline levels.

(5) All extraction operations must be as short as possible and carried out by an experienced veterinarian to minimize over-sensitization of the musk sac wall.

Acknowledgement: We thank Cunxuan Li, Libin Li, and Deyun Yang for their valuable suggestions and help on sample collection. Special thanks to all the breeders of the Shanxi Pien Tze Huang Forest Musk Deer Breeding Center. This work received financial support from Fundamental research funds for the central universities (Grant No. 2019YC18), National Key R\&D Program of the P.R. of China (2018YFD0502204-1) and National Natural Science Foundation of China (NSFC)(31872962).

Ethics approval: This study was approved by the Ethics Committee of Beijing Forestry University, Beijing, China; Pien Tze Huang Pharmaceutical Corporation, Zhangzhou, China; and Pien Tze Huang Forest Musk Deer Breeding Center, Shaanxi, China. This study was also carried out in accordance with the recommendations of the Institution of Animal Care and the Ethics Committee of Beijing Forestry University. All experimental procedures were performed with the help of an expert veterinarian, and all efforts were made to minimize suffering.

Authors' contributions: YS and MZ designed the study and carried out the experiments, YL and MC participated in sample collection and experiments, SX, TZ and YL participated in performing ELISA tests, XG, MS and SL 
conducted the data analysis, DH and TW prepared and revised the manuscript.

\section{REFERENCES}

Becker, B. G., and J. P. Lobato (1997). Effect of gentle handling on the reactivity of zebu crossed calves to humans. Appl. Anim. Behav. Sci. 53(3): 219224.

Bonesi, L., and S. Palazon (2007). The american mink in europe: Status, impacts, and control. Biol. Conserv. 134(4): 470-483.

Brown, G., and D. F. Layton (2001). A Market Solution for Preserving Biodiversity: The Black Rhino. In: Protecting Endangered Species in the United States: Biological Needs, Political Realities, Economic Choices; Shogren, J.F. and Tschirhart, J. (Eds.). Cambridge University Press, Cambridge, p. 32-50.

Buenviaje, G., P. Ladds, L. Melville, and S. Manolis (1994). Disease-husbandry associations in farmed crocodiles in Queensland and the Northern Territory. Aust. Vet. J. 71(6): 165-173.

Bulte, E. H., and R. Damania (2005). An Economic Assessment of Wildlife Farming and Conservation. Conserv. Biol. 19(4): 1222-1233.

Capezzuto, A., M. O. Chelini, E. C. Felippe, and C. A. Oliveira (2008). Correlation between serum and fecal concentrations of reproductive steroids throughout gestation in goats. Anim. Reprod. Sci. 103(1-2): 78-86.

Cheng, J., Y. Luo, M. Qiao, Y. Cai, and D. Wang (2002). The factors affecting musk secretion quantity in Moschus berezovskii. Special Wild Economic Animal and Plant Research 3: 15-18.

Crookshank, H. R., M. H. Elissalde, R. G. White, D. C. Clanton, and H. E. Smalley (1979). Effect of transportation and handling of calves upon blood serum composition. J. Anim. Sci. 48(3): 430.

Cyr, N. E., and L. Michael Romero (2007). Chronic stress in free-living European starlings reduces corticosterone concentrations and reproductive success. Gen. Comp. Endocrinol. 151(1): 82-89.

Davis, A. K., D. L. Maney, and J. C. Maerz (2008). The use of leukocyte profiles to measure stress in vertebrates: a review for ecologists. Funct. Ecol. 22(5): 760-772.

Dhabhar, F. S., and B. S. McEwen (1997). Acute stress enhances while chronic stress suppresses cellmediated immunityin vivo: A potential role for leukocyte trafficking. Ann. N. Y. Acad. Sci. 11(4): 286-306.

Foley, K.-E., C. J. Stengel, and C. R. Shepherd (2011). Pills, powders, vials and flakes: The bear bile trade in Asia. TRAFFIC Southeast Asia, Petaling Jaya, Selangor, Malaysia.
Franceschini, M. D., D. I. Rubenstein, B. Low, and L. M. Romero (2008). Fecal glucocorticoid metabolite analysis as an indicator of stress during translocation and acclimation in an endangered large mammal, the Grevy's zebra. Anim. Conserv. 11(4): 263-269.

Gouin, J. P., L. Hantsoo, and J. K. Kiecolt-Glaser (2008). Immune dysregulation and chronic stress among older adults: a review. Neuroimmunomodulation 15(4-6): 251-259.

Hargreaves, A. L., and G. D. Hutson (1990). Changes in heart rate, plasma cortisol and haematocrit of sheep during a shearing procedure. Appl. Anim. Behav. Sci. 26(1): 91-101.

Hassanin, A., F. Delsuc, A. Ropiquet, C. Hammer, B. Jansen van Vuuren, C. Matthee, M. Ruiz-Garcia, F. Catzeflis, V. Areskoug, T. T. Nguyen, and A. Couloux (2012). Pattern and timing of diversification of cetartiodactyla (Mammalia, Laurasiatheria), as revealed by a comprehensive analysis of mitochondrial genomes. C. R. Biol. 335(1): 32-50.

He, L., W. X. Wang, L. H. Li, B. Q. Liu, G. Liu, S. Q. Liu, L. Qi, and D. F. Hu (2014). Effects of crowding and sex on fecal cortisol levels of captive forest musk deer. Biol. Res. 47(1): 48.

Hemsworth, P. H., J. L. Barnett, and C. Hansen (1987). The influence of inconsistent handling by humans on the behaviour, growth and corticosteroids of young pigs. Appl. Anim. Behav. Sci. 17(3): 245-252.

Homes, V. (1999). On the scent: Conserving musk deer: The uses of musk and europe's role in its trade. Traffic Europe, Brussels.

Isobe, N., M. Akita, T. Nakao, H. Yamashiro, and H. Kubota (2005). Pregnancy diagnosis based on the fecal progesterone concentration in beef and dairy heifers and beef cows. Anim. Reprod. Sci. 90(3-4): 211-218.

IUCN (2018). The IUCN Red List of Threatened Species. Available from: https://www.iucnredlist.org.

Kang, F. G., R. T. Zhao, and Z. X. Liu (2008). The research on obtaining musk from the living body of alpine musk deer and its production in the farm. J. Gansu Sciences 20(2): 96-98.

Kralj-Fišer, S., B. M. Weiß, and K. Kotrschal (2009). Behavioural and physiological correlates of personality in greylag geese (Anser anser). J. Ethol. 28(2): 363-370.

Lang, D., W. Liu, D. Hu, Y. Wang, and X, Ge (2011). Evaluation of the storage of fecal samples of captive forest musk deer for steroid analysis. Sichuan J. Zool. 30(03):357-361.

Laule, G. E., M. A. Bloomsmith, and S. Schapiro (2003). The use of positive reinforcement training techniques to enhance the care, management, 
and welfare of primates in the laboratory. J. Appl. Anim. Welf. Sci. 6(3): 163-173.

Linklater, W., E. MacDonald, J. Flamand, and N. Czekala (2010). Declining and low fecal corticoids are associated with distress, not acclimation to stress, during the translocation of african rhinoceros. Anim. Conserv. 13(1): 104-111.

McEwen, B. S. (2004). Protection and damage from acute and chronic stress: Allostasis and allostatic overload and relevance to the pathophysiology of psychiatric disorders. Ann. N. Y. Acad. Sci. 1032(1): 1-7.

Meng, Q. F., Y. Li, Y. Zhou, Y. D. Bai, W. L. Wang, W. L. Wang, and W. Cong (2015). Seroprevalence of neospora caninum infection in farmed sika deer (Cervus nippon) in China. Vet. Parasitol. 211(34): 289-292.

Meng, X., B. Gong, G. Ma, and L. Xiang (2011a). Quantified Analyses of Musk Deer Farming in China: A Tool for Sustainable Musk Production and Ex situ Conservation. Asian-Australas. J. Anim. Sci. 24(10): 1473-1482.

Meng, X., C. Zhao, C. Hui, and X. Luan (2011b). Behavioral Aspects of Captive Alpine Musk Deer during Non-mating Season: Gender Differences and Monthly Patterns. AsianAustralas. J. Anim. Sci. 24(5): 707-712.

Meng, X. X., H. Yang, Q. S. Yang, Z. J. Feng, X. Peng, and G. C. Perkins (2010). Preliminary findings of behavioral patterns in captive alpine musk deer (Moschus sifanicus) and prospects for future conservation. Turk. J. Vet. Anim. Sci. 34(2): 111-117.

Miller, G. E., E. Chen, and E. S. Zhou (2007). If it goes up, must it come down? Chronic stress and the hypothalamic-pituitary-adrenocortical axis in humans. Psychol. Bull. 133(1): 25.

Millspaugh, J. J., T. Burke, G. U. S. Van Dyk, R. O. B. Slotow, B. E. Washburn, and R. J. Woods (2007). Stress response of working african elephants to transportation and safari adventures. J. Wildl. Manage. 71(4): 1257-1260.

Mitra, B. (2005). How the market can save the tiger. FEER. 168: 44-47.

Moberg, G. P. (1985). Biological response to stress: Key to assessment of animal well-being? Animal Stress. Springer, New York, NY. p. 27-49.

Morato, R. G., M. G. Bueno, P. Malmheister, I. T. Verreschi, and R. C. Barnabe (2004). Changes in the fecal concentrations of cortisol and androgen metabolites in captive male jaguars (Panthera onca) in response to stress. Braz. J. Med. Biol. Res. 37(12): 1903-1907.

Moreira, N., E. L. Monteiro-Filho, W. Moraes, W. F. Swanson, L. H. Graham, O. L. Pasquali, M. L. Gomes, R. N. Morais, D. E. Wildt, and J. L.
Brown (2001). Reproductive steroid hormones and ovarian activity in felids of the Leopardus genus. Zoo. Biol. 20(2): 103-116.

Morgan, K. N., and C. T. Tromborg (2007). Sources of stress in captivity. Appl. Anim. Behav. Sci. 102(3-4): 262-302.

Möstl, E., and R. Palme (2002). Hormones as indicators of stress. Domest. Anim. Endocrinol. 23(1): 67 74

Narayan, E., and J. M. Hero (2011). Urinary corticosterone responses and haematological stress indicators in the endangered Fijian ground frog (Platymantis vitiana) during transportation and captivity. Aust. J. Zool. 59(2): 79-85.

Nephew, B. C., and L. M. Romero (2003). Behavioral, physiological, and endocrine responses of starlings to acute increases in density. Horm. Behav. 44(3): 222-232.

Orihuela, A., V. Aguirre, C. Hernandez, I. Floresperez, and R. Vazquez (2012). Effect of anesthesia on welfare aspects of hair sheep (Ovis aries) during electro-ejaculation. J. Anim. Vet. Adv. 8(2): 305308.

Palme, R., C. Robia, W. Baumgartner, and E. Mostl (2000). Transport stress in cattle as reflected by an increase in faecal cortisol metabolite concentrations. Vet. Rec. 146(4): 108-109.

Parry-Jones, R., and J. Y. Wu (2001). Musk deer farming as a conservation tool in China. TRAFFIC East Asia, Hong Kong, China.

Pierzchala, K., S. Bobek, J. Niezgoda, and Z. Ewy (1983). The effect of shearing on the concentration of cortisol and thyroid hormones in the blood plasma of sheep. Zentralbl. Veterinarmed. A. 30(10): 749-759.

Pilz, W. (1997). Der moschusduft-eine parfumhistorische betrachtung. SEPAWA Kongreßzeitschrift, Verlag für chemische Industrie, H. Ziolkowsky GmbH, Augsburg, Germany, p. 43-47.

Prunier, A., A. M. Mounier, and M. Hay (2005). Effects of castration, tooth resection, or tail docking on plasma metabolites and stress hormones in young pigs. J. Anim. Sci. 83(1): 216-222.

Revol, B. (1995). Crocodile farming and conservation, the example of Zimbabwe. Biodivers. Conserv. 4(3): 299-305.

Romero, L. M., and J. M. Reed (2005). Collecting baseline corticosterone samples in the field: Is under $3 \mathrm{~min}$ good enough? Comp. Biochem. Phys. A. 140(1): 73-79.

Sheng, H. L., and Z. X. Liu (2007). The musk deer in China. The Shanghai Scientific \& Technical Publishers, Shanghai.

Shrestha, M. (1998). Animal welfare in the musk deer. Appl. Anim. Behav. Sci. 59(1-3): 245-250.

Shrestha, M. N. (1989). Musk deer Moschus chrysogaster 
musk extraction from live deer. J. Bombay Nat. Hist. Soc. 86: 438-440.

Smith, J. D., S. W. Allen, and J. E. Quandt (1999). Changes in cortisol concentration in response to stress and postoperative pain in client-owned cats and correlation with objective clinical variables. Am. J. Vet. Res. 60(4): 432-436.

Teixeira, C. P., C. S. De Azevedo, M. Mendl, C. F. Cipreste, and R. J. Young (2007). Revisiting translocation and reintroduction programmes: the importance of considering stress. Anim. Behav. 73(1): 1-13.

Tensen, L. (2016). Under what circumstances can wildlife farming benefit species conservation? Glob. Ecol. Conserv. 6: 286-298.

Thompson, V. D. (1989). Behavioral response of 12 ungulate species in captivity to the presence of humans. Zoo Biol. 8(3):275-297.

UNEP-WCMC (2014). Checklist of cites species. CITES Secretariat, Geneva, Switzerland and UNEPWCMC, Cambridge, UK. [accessed 02 June 2020].

Wada, K., M. Nishibori, and M. Yokohama (2007). The complete nucleotide sequence of mitochondrial genome in the japanese sika deer (Cervus nippon), and a phylogenetic analysis between cervidae and bovidae. Small Ruminant Res. 69(1-3): 46-54.

Wang, L., H. Wang, H. P. Liu, J. D. Liu, H. Ning, Q. Y. Wang, Z. Q. Cheng, and Y. J. Wang (2015). Breeding and development of musk deer in sichuan. Pharmacology and Clinics of Chinese Materia Medica 6(4): 6.

Wang, W., L. He, S. Liu, T. Wronski, and D. Hu (2016). Behavioral and physiological responses of forest musk deer (Moschus berezovskii) to experimental fawn manipulation. Acta Ethol.
19(2): 133-141.

Wasser, S. K., L. Risler, and R. A. Steiner (1988). Excreted steroids in primate feces over the menstrual cycle and pregnancy. Biol. Reprod. 39(4): 862-872.

Weinberg, J., and S. Levine (1980). Psychobiology of coping in animals: The effects of predictability. Coping and Health 12: 39-59.

Wells, A., K. A. Terio, M. H. Ziccardi, and L. Munson (2004). The stress response to environmental change in captive cheetahs (Acinonyx jubatus). J. Zoo Wildl. Med. 35(1): 8-14.

Wielebnowski, N. C., N. Fletchall, K. Carlstead, J. M. Busso, and J. L. Brown (2002). Noninvasive assessment of adrenal activity associated with husbandry and behavioral factors in the north american clouded leopard population. Zoo Biol. 21(1): 77-98.

Wu, J. Y., and W. Wang (2006). The musk deer of China. China Forestry Publishing House, Beijing.

Yang, Q., X. Meng, L. Xia, and Z. Feng (2003). Conservation status and causes of decline of musk deer (Moschus spp.) in China. Biol. Conserv. 109(3): 333-342.

Yu, X., Hu, D., Jin, X., Ge, X., Yang, L., Zhao, P., and Q. Zhang (2011). Non-invasive determination of fecal steroid hormones relating to conservation practice in giant panda (Ailuropoda melanoleuca). Anim. Biol. 61(3): 335-347.

Zhang, B. L. (1983). Musk-deer: their capture, domestication and care according to chinese experience and methods. Unasylva 35: 16-24.

Zhang, H. Z., M. Wang, J. Y. Li, J. B. Shao, and Y. T. Ma (2009). Living Extraction of Musk from Moschus berezovskii in Captivity. Chinese J. Wildlife 30(4): 175-176. 